\title{
An Analysis of Star Topology IEEE 802.11e Networks in the Presence of Hidden Nodes
}

\author{
Katarzyna Kosek ${ }^{1}$, Marek Natkaniec ${ }^{1}$, Luca Vollero ${ }^{2}$, Andrzej R. Pach ${ }^{1}$, Members, IEEE \\ ${ }^{1}$ AGH-University of Science and Technology, Department of Telecommunications, Poland \\ ${ }^{2}$ Consorzio Interuniversitario Nazionale per l'Informatica - University of Napoli, Italy \\ \{kkosek, natkaniec, vollero\}lieee.org, pach@kt.agh.edu.pl
}

\begin{abstract}
This article presents a novel simulation study of IEEE 802.11e based environments. The most important innovation of this article lays in demonstrating a thorough analysis of two star topology networks, which shows how seriously they can be degraded by the presence of hidden nodes. The main stress is put on the limitation of the ability to provide services with the requested QoS. The usefulness of the RTS/CTS mechanism is also discussed. According to the authors' best knowledge, similar studies have never been made. ${ }^{1}$
\end{abstract}

\section{INTRODUCTION}

Especially now, when the user demands for bandwidth are constantly growing and when the number of such delay sensitive services as VoIP, VoD or multimedia streaming is increasing rapidly, proper quality of service (QoS) assurance is crucial. However, despite many years of research, providing QoS guarantees within wireless networks remains an unsolved problem. This challenge is even more complicated when we focus on ad hoc networks as their topology and traffic load change frequently in a completely unpredictable way. Within such networks, due to their nature, hidden nodes are likely to appear and cause serious degradation of their performance.

The presented novel analysis of two different 802.11e network topologies reveals surprising results and helps to draw many innovative conclusions. It shows serious unfairness in granting medium access and, furthermore, it demonstrates how significantly the expected throughput levels of different priority traffic streams are distorted when the hidden terminal problem appears. Additionally, this paper argues the usefulness of the RTS/CTS mechanism and points out its main limitations. Furthermore, also the hitherto unnoticed flaws of the MAC enhancements proposed in the IEEE 802.11e standard are stressed.

The rest of the paper is organized as follows. Section 2 explains the hidden terminal problem. In Section 3 the IEEE 802.11e standard is briefly described. Section 4 and Section 5 give a detailed description of the analyzed scenarios and discuss the achieved results, respectively. The summarizing conclusions can be found in Section 6 .

\section{Hidden TERMinAl PROBLEM}

One of the meaningful disadvantages of the wireless nodes is their half-duplex nature which prevents simultaneous reception and transmission of data. As a result, when two or

\footnotetext{
${ }^{1}$ This work has been carried out under the UE-funded NoE CONTENT project no. 038423
}

more stations start their transmissions at the same time a collision is unavoidable. Due to the fact that the CSMA/CA procedure is unable to detect hidden nodes an optional four way handshake mechanism was proposed to improve the performance of 802.11 environments. During its operation two extra frame types are exchanged when granting medium access, namely, RTS (Request-To-Send) and CTS (Clear-ToSend).

In the literature several concurrent solutions to the RTS/CTS mechanism can be found, however, none of them has become broadly popular and, usually, it is the four way handshake mechanism which is selected to combat the hidden terminal problem. Brief descriptions of the most important examples of different MAC protocols can be found in [3]. The usefulness and main limitations of RTS/CTS have been discussed in a number of papers (e.g., [6][7][8]), however, none of them was related to the matters presented in this paper (i.e., RTS/CTS in 802.11e with hidden stations).

\section{802.11E BACKGROUND}

The IEEE 802.11e standard defines two modes - EDCA (Enhanced Distributed Channel Access) and HCCA (Hybrid Coordination function Channel Access). In view of the fact that EDCA is used by the ad hoc networks it is described in more details next. For more details on HCCA see [1].

The EDCA function defines several extensions to the traditional CSMA/CA procedure, which is as distributed wireless medium access method. Its main aim it to assure the transportation of traffic with a desired QoS. EDCA introduces four access categories (ACs) characterized by several different access parameters, associated with four independent priorities. Since voice $(\mathrm{Vo})$ and video (Vi) traffic is more jitter, delay and packet loss sensitive it has a higher priority than best effort (BE) and background (BK) traffic (Fig. 1).

The process of channel access prioritization is depicted in Fig. 1 and Fig. 2 and can be briefly described as follows. Inside a QoS node, on the basis of its type, each frame of a particular traffic stream is mapped to an appropriate AC. Then, it is buffered into one of four hardware transmission queues. After that, the probability of being granted the channel access is strictly dependent on four access parameters, i.e., an arbitrary interfame space (AIFSN[AC]), contention window minimum and maximum boundary limits $\left(C W_{\min }[A C]\right.$ and $\left.C W_{\max }[A C]\right)$, and an optional transmission opportunity duration limit $\left(T X O P_{\text {limit }}\right)$. On the basis of these access parameters the values of the backoff interval and AIFS are 
computed for each of the ACs. The backoff value is set to a random number from the number interval $[0, \mathrm{CW}]$, where $\mathrm{CW}$ initially equals $C W_{\min }[A C]$ and is increased up to $C W_{\max }[A C]$ whenever the $\mathrm{AC}$ involves in a collision. AIFS[AC] is given by equation:

$$
A I F S[A C]=S I F S+A I F S N[A C] \times \text { SlotTime }
$$

A QoS node is assigned the right to transmit every time when the medium was sensed idle for AIFS[AC] and when the backoff time for a particular AC has elapsed. As an obvious consequence, the smaller the AIFSN[AC] and the contention window size, the higher the probability of being granted earlier access to the wireless medium.

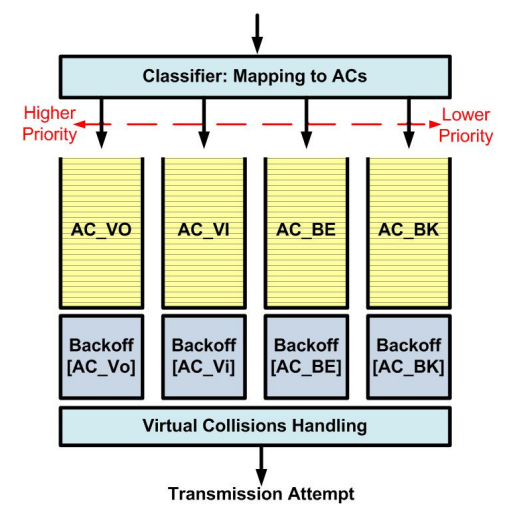

Fig. 1 Virtual backoff procedure

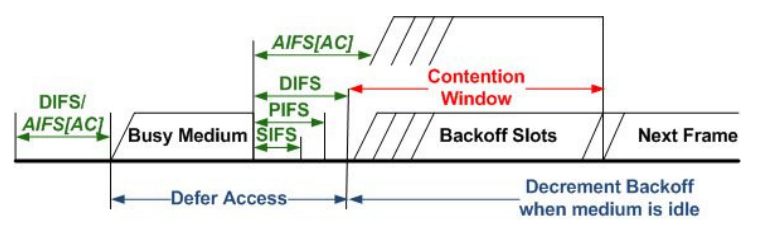

Fig. 2 Channel access prioritization [1]

Two types of collisions may occur during the EDCA channel access procedure. Firstly, a virtual collision appears every time when more than one AC within a node is granted the right to transmit at the same time. Secondly, a physical collision happens every time when two or more QoS nodes start their transmissions over the wireless medium simultaneously.

\section{IV.SCENARIOS}

The simulation analysis was performed with the use of the ns2 simulator and an improved version of the TKN 802.11e EDCA model [3]. The changes made affect the RTS/CTS mechanism and were validated with the use of the OPNET [5] simulator. The common 802.11b PHY [2], EDCA and other simulation parameters are given in Table 1 and Table 2. TXOP $_{\text {limit }}$ was not used, therefore, it is not included in Table 2.

For every analyzed network setup, we simulated four different EDCA configurations. In each configuration a different EDCA class is used for the flows generated by the nodes. Moreover all nodes send the same traffic type with a varying sending rate (from $10 \mathrm{~kb} / \mathrm{s}$ to $5 \mathrm{Mb} / \mathrm{s}$ for the five-node star and from $10 \mathrm{~kb} / \mathrm{s}$ to $7 \mathrm{Mb} / \mathrm{s}$ for the four-node star). The plots present the curves, where the error of each simulation point for a $95 \%$ confidence interval does not exceed $2 \%$.
TABLE 1

COMMON SIMULATION PARAMETERS

\begin{tabular}{|l|l|l|l|}
\hline \hline SIFS & $10 \mu \mathrm{s}$ & DIFS & $50 \mu \mathrm{s}$ \\
\hline PIFS & $30 \mu \mathrm{s}$ & Slot Time & $20 \mu \mathrm{s}$ \\
\hline Tx Range & $250 \mathrm{~m}$ & Tx Power & $0.282 \mathrm{~W}$ \\
\hline Frame Size & $1000 \mathrm{~B}$ & $\begin{array}{l}\text { Traffic } \\
\text { Type }\end{array}$ & CBR \\
\hline Carrier Sensing Range & $263 \mathrm{~m}$ & Distance & $200 \mathrm{~m}$ \\
\hline Wireless Standard & IEEE 802.11b with 802.11e \\
\hline \hline
\end{tabular}

TABLE 2

EDCA PARAMETER SET

\begin{tabular}{|l|l|l|l|l|}
\hline \hline Priority & AC & $\boldsymbol{C W}_{\min }[\boldsymbol{A C}]$ & $\boldsymbol{C} \boldsymbol{W}_{\max }[\boldsymbol{A C}]$ & $\boldsymbol{A I F S N [ A C ]}$ \\
\hline P0 & Vo & 7 & 15 & 2 \\
\hline P1 & Vi & 15 & 31 & 2 \\
\hline P2 & BE & 31 & 1023 & 3 \\
\hline P3 & BK & 31 & 1023 & 7 \\
\hline \hline
\end{tabular}

\section{A. Scenario 1: four-node star}

The first of the two analyzed scenarios consists of four nodes from which three are hidden from each other (Fig. 3). All of the hidden nodes are constantly sending CBR traffic to the unhidden Node 1 (N1). N1 is transmitting to Node 4 (N4). In all simulations packet generation times of different nodes are not synchronized.

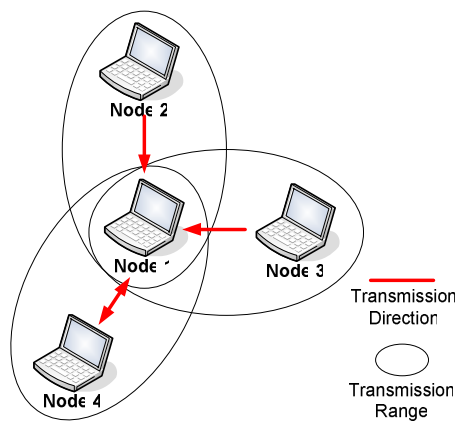

Fig. 3 Scenario 1 - four node star topology IEEE 802.11e network

\section{B. Scenario 2: five-node star}

The second scenario consisted of five nodes from which four are hidden (Fig. 4). The hidden nodes are continuously sending data to N1, while, N1 is transmitting to N5.

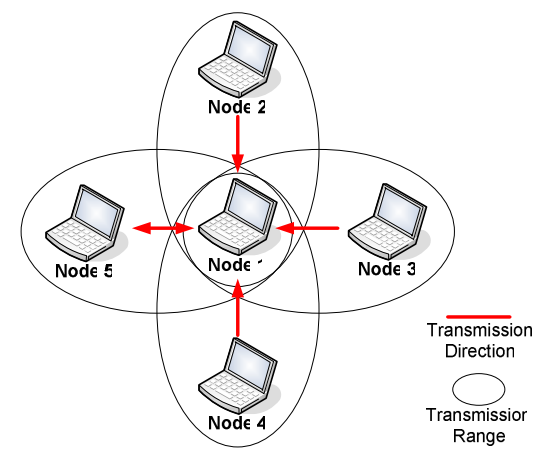

Fig. 4 Scenario 2 - five node star topology IEEE 802.11e network

Despite of the fact that Scenario 2 seems similar to Scenario 1 it helps in formulation of a meaningful conclusion which will be described in the following section in more detail. 


\section{Simulation Results}

\section{A. Scenario 1}

In Fig. 5 and Fig. 6, the curves of different traffic priorities are shown. They represent the obtained throughput as a function of the total offered load. Fig. 5 depicts the situation in which the RTS/CTS exchange was disabled, Fig. 6 - the situation in which RTS/CTS was enabled. Furthermore, due to the fact that the hidden nodes gained almost the same throughput, for the clarity of presentation, only their mean throughput is shown and is denoted as HA (Hidden Average). The throughput of N1 was different; therefore, it is presented separately and denoted as N1.

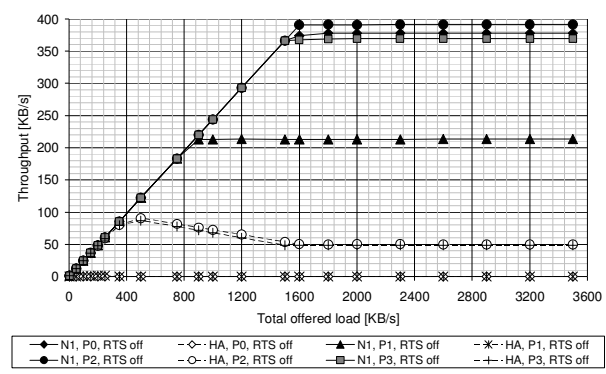

Fig. 5 Scenario 1, RTS/CTS disabled

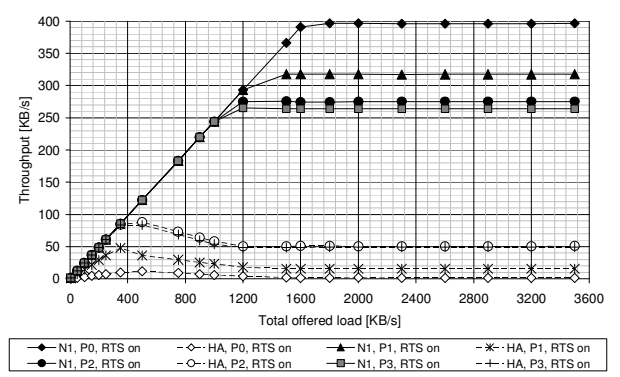

Fig. 6 Scenario 1, RTS/CTS enabled

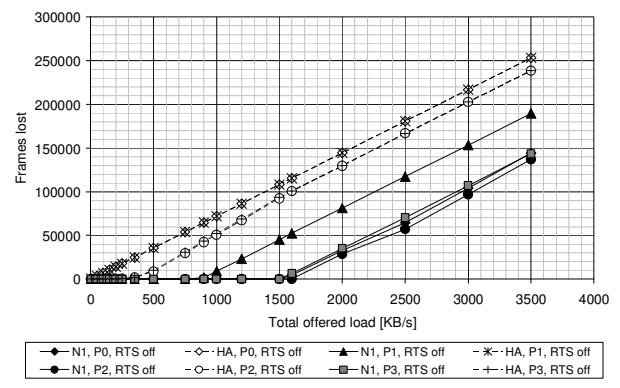

Fig. 7 Scenario 1: the frame loss, RTS/CTS disabled

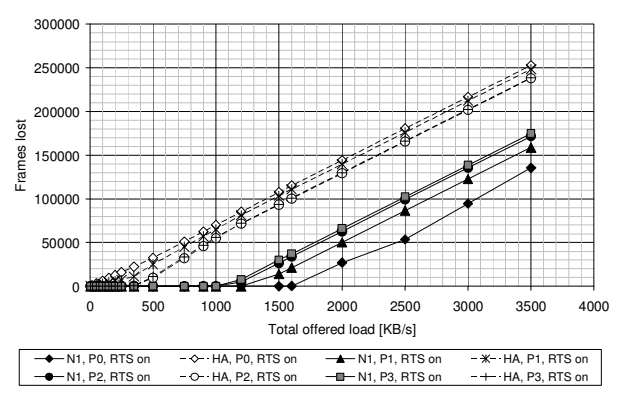

Fig. 8 Scenario 1: the frame loss, RTS/CTS enabled
Fig. 7 and Fig. 8 show the total number of lost frames as a function of the total offered load with enabled and disabled RTS/CTS, respectively. The separate figures for interface queue drops, retransmissions, and collisions are not included due to the lack of space.

The most significant conclusions from the obtained results are as follows. With disabled RTS/CTS, hidden stations sending $\mathrm{BE}$ and $\mathrm{BK}$ traffic have significantly better throughput than the ones sending Vi and Vo. Moreover, the throughput levels observed for the unhidden node $\mathrm{N} 1$ are seriously disordered, i.e., BE and BK have better throughput than Vi. With both, disabled and enabled RTS/CTS, N1 has considerably better throughput than any other station of all traffic classes. When the RTS/CTS mechanism is enabled the order of the throughput levels for $\mathrm{N} 1$ are in line with the 802.11e guidelines. Unfortunately, the throughput values achieved by the hidden nodes are still unsatisfactory as enabling RTS/CTS resulted only in a hardly noticeable increase of Vo and Vi throughput. In order to understand this unexpected performance, the following four questions can be posed.

1) In Fig. 5: Why is the throughput of $N 1$, regarding the traffic priorities, as follows: $P 2>P 0>P 3>P 1$ ?

Such an order is caused by the varied number of MAC interface queue drops (ifq drops, i.e., frames dropped due to the overflow of the interface queue between the Link Layer and the MAC Layer) observed for different traffic priorities. The number of collisions and, thus, retransmissions practically equals zero thanks to the central (unhidden) placement of N1. As a consequence, the concatenated number of lost frames is determined only by the ifq drops and can be seen in Fig. 7 . Additionally, the slight differences in the throughput levels experienced by P0, P2 and P3 can be explained by the values of their EDCA parameters which impact the prioritization in the medium access.

2) In Fig. 5: Why is the throughput of HA as follows: $P 2 \geq P 3>P 0=P 1$ ?

Due to the fact that the number of ifq drops is practically equal to all traffic priorities only the number of collisions and, therefore, retransmissions cause differentiation in the general frame loss (Fig. 7). P0 experienced the highest number of collisions and retransmissions, P1 slightly lower, while P3 and P2 the lowest.

3) In Fig. 6: Why is the throughput of N1 as follows: $P 0>P 1>P 2>P 3$ ?

Obviously, the position of $\mathrm{N} 1$ causes the number of collisions and retransmissions practically equal to zero. The total frame loss is determined by the number of ifq drops (Fig. 8) which is highest for P3, lower for P2, a bit lower for P1 and lowest for P0. Consequently, the order of frame loss is in order with the 802.11e guidelines.

4) In Fig. 6: Why is the throughput of HA as follows: $P 2 \geq P 3>P 1>P 0$ ? 
The summed number of lost frames is shown in Fig. 8. It is determined by the number of retransmissions as the number of ifq drops is practically equal for all priorities and the number of collisions is reduced to zero. The number of retransmissions is highest for $\mathrm{P} 0$, smaller for $\mathrm{P} 1$, the smallest (and equal) for P2 and P3.

The explanations of the posed questions lead to several important conclusions. First of all, the higher the priority the more often the stations compete for medium access $\left(C W_{\min }\right.$ and $C W_{\max }$ values are smaller than for low priority traffic) and, as a result, the hidden nodes experience more collisions and retransmissions while the unhidden node has more ifq drops. The reason why the hidden nodes lose frames due to collisions and, therefore, retransmissions is simple - if there are 3 nodes hidden from each other which try to gain medium access frequently, there is a higher probability that one station will try to transmit its data at exactly the same time as others. At the same time, N1 can hear other stations' transmissions and collisions; therefore, instead of sending data frames it will keep them in its priority queues. Obviously, the quicker the priority queues will be overloaded (which, in the scenario with disabled RTS/CTS, happens for high priority traffic) - the higher the number of frames will be dropped. As the number of collisions counted for the hidden stations decreases to zero with enabled RTS/CTS, N1 has more chances to send its data (in particular, its high priority traffic). Thus, in comparison to Fig. 5, the throughput levels shown in Fig. 6 meaningfully decrease for P3 and P2 and increase for P1 and P0. Moreover, this causes their order to be in line with the 802.11e guidelines. Another surprising fact may be the high number of collisions of $\mathrm{Vi}$ and $\mathrm{Vo}$ traffic for the hidden nodes with a poor network load. This situation is present for both enabled and disabled RTC/CTS. It is a result of a peculiar synchronization pattern. Due to the long frame sizes $(1000 \mathrm{~B})$ and a single time slot value of $20 \mu \mathrm{s}$, more than 36 time slots are needed (in 802.11b) for an undisturbed transmission. Unfortunately, for $\mathrm{Vo}$ and $\mathrm{Vi}$ traffic the maximum $\mathrm{CW}$ size equals 15 and 31 , respectively. Therefore, this unacceptable number of collisions is unavoidable. The simplest antidote to this problem would be an obligatory process of frame fragmentation.

\section{B. Scenario 2}

Fig. 9 and Fig. 10 depict the curves which represent the obtained throughput as a function of the total offered load achieved by the different traffic priorities. They show the situation with disabled and enabled RTS/CTS, respectively. Furthermore, similarly to Scenario 1, for the hidden nodes only their mean throughput is presented (HA), while the throughput of $\mathrm{N} 1$ is shown separately.

Correspondingly to Scenario 1, Fig. 11 and Fig. 12 show the total number of the lost frames as a function of the total offered load with enabled and disabled RTS/CTS, respectively.

The most significant conclusions from the obtained results are as follows. When the four way handshake mechanism is disabled, stations sending lower priority traffic (BE and BK) have meaningfully better throughput than the ones sending high priority traffic ( $\mathrm{Vi}$ and $\mathrm{Vo}$ ), regardless whether they are

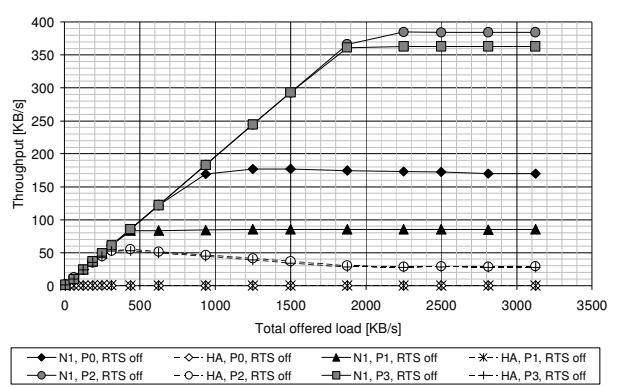

Fig. 9 Scenario 2, RTS/CTS disabled

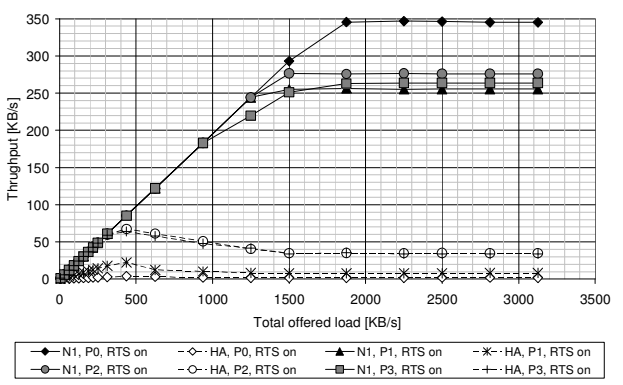

Fig. 10 Scenario 2, RTS/CTS enabled

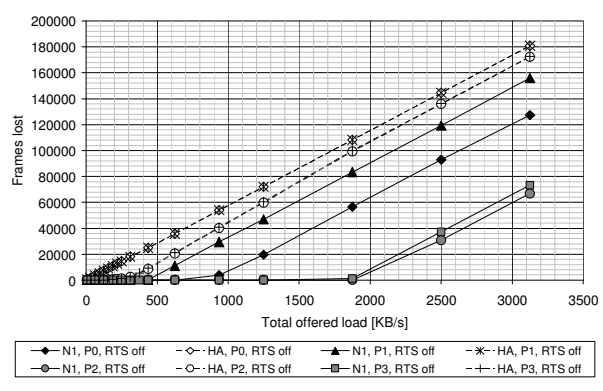

Fig. 11 Scenario 2: the frame loss, RTS/CTS disabled

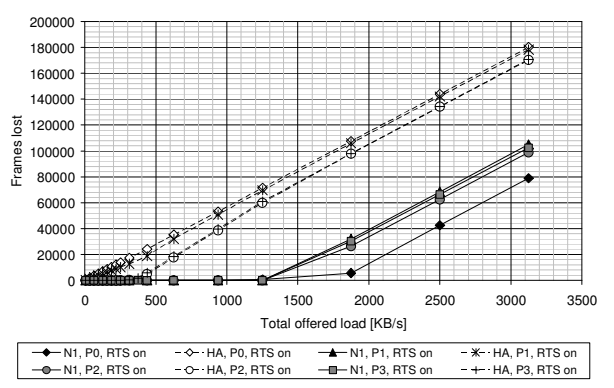

Fig. 12 Scenario 2: the frame loss, RTS/CTS enabled

hidden or not. Moreover, N1, in both cases, has significantly better throughput than any other station for every traffic class. Additionally, when the RTS/CTS exchange is enabled the desired results are still not obtained. From the achieved results it is clearly noticeable that, although the fact that the throughput gained by $\mathrm{N} 1$ sending $\mathrm{BE}$ and $\mathrm{BK}$ traffic decreased and, at the same time, increased for $\mathrm{Vi}$ and $\mathrm{Vo}$, the throughput achieved by the hidden stations is still dramatically low. Furthermore, the levels of the obtained throughput for different traffic classes are totally disordered. For the unhidden station $\mathrm{N} 1$ the throughput observed for $\mathrm{Vi}$ is lower than the ones observed for $\mathrm{BE}$ and $\mathrm{BK}$. For the hidden nodes 
the situation is even worse as both $\mathrm{Vi}$ and $\mathrm{Vo}$ obtained much lower throughput than BE and BK. Additionally, all of the throughput levels are different than observed in the four-node star scenario for both enabled and disabled RTS/CTS. Especially for $\mathrm{N} 1$ their order is meaningfully worse and is much farther from the 802.11e expectations. For the hidden nodes the obtained throughput is even more unfavorable than in the previous scenario. In order to explain this surprising performance the following four questions can be posed.

1) In Fig. 9: Why is the throughput of N1, as follows: $P 2>P 3>P 0>P 1$ ?

It is caused mostly by the fact that the number of ifq drops is highest for P1, slightly lower for P0, much lower for P3 and lowest for P2. In this situation ifq drops took a dominant percentage of the frame loss and, therefore, the curves representing the total number of the lost frames have similar trends (Fig. 11).

2) In Fig. 9: Why is the throughput of HA: $P 2=P 3>P 0=P 1$ ?

The number of ifq drops for P1 and P0 are almost equal and slightly smaller than the similar number of ifq drops observed for P3 and P2. The number of collisions and, therefore, retransmissions for $\mathrm{P} 0$ and $\mathrm{P} 1$ is much higher than the corresponding number for $\mathrm{P} 3$ and $\mathrm{P} 2$. As a result, the overall frame loss for P0 and P1 is higher than the one observed for P3 and P2 (Fig. 11).

3) In Fig. 10: Why is the throughput of N1 as follows: $P 0>P 2>P 3>P 1$ (in saturation)?

It is because the number of ifq drops for P1 is a little bit higher than the number of ifq drops for P3. At the same time, the number of ifq drops counted for P0 is slightly lower than the number of ifq drops for P2 and much lower than the number observed for P1 and P3. Obviously, the number of collisions and retransmissions of $\mathrm{N} 1$ practically equals zero. The total number of the lost frames is shown in Fig. 12.

4) In Fig. 10: Why is the throughput of HA as follows: $P 2 \geq P 3>P 1>P 0$ ?

The number of ifq drops for $\mathrm{P} 1$ and $\mathrm{P} 0$ are almost equal and smaller than the comparable number of ifq drops for P3 and $\mathrm{P} 2$. The number of retransmissions for $\mathrm{P} 1$ is higher than for P0 and is much lower than for P3 and P2. The number of DATA collisions for all traffic priorities practically equals zero. The general frame loss is similar for P0 and P1 (for P0 it is only slightly higher) and is higher than the one observed for, almost identical, P3 and P2 (Fig. 12).

On the basis of the answer to the above questions and the results obtained for the first scenario general conclusions can be drawn - with higher priorities the hidden nodes experience more collisions and, therefore, retransmissions while N1 experiences more ifq drops. Additionally, the number of these frame loss types increases with the increase in the ratio of the hidden to unhidden nodes. Consequently, the throughput levels and their order are much worse. Another conclusion is that the quicker the priority queues are overloaded the higher number of frames is dropped. Therefore, similarly to Scenario 1, also in Scenario 2 with enabled RTS/CTS, the number of collisions counted for the hidden stations decreases to zero and N1 has more chances to send its high priority traffic. As shown in Fig. 10, the throughput of N1 meaningfully decreased for P3 and P2 while increased for P1 and P0 in comparison to the network with disabled RTS/CTS (see Fig. 9). For P3 and P2 it decreased around $100 \mathrm{~KB} / \mathrm{s}$ and for $\mathrm{P} 1$ and $\mathrm{P} 0$ it increased around $170 \mathrm{~KB} / \mathrm{s}$. However, in this scenario, this does not bring the order of the throughput levels in line with the 802.11e guidelines. Obviously, the problem of the odd synchronization is present in Scenario 2 which causes an unacceptable number of collisions. The final overall conclusions are that the medium access is meaningfully unfair when hidden stations appear in a particular network and, additionally, when the number of hidden stations increases the order of throughput levels is more distorted.

\section{CONCLUSIONS}

This paper presents a simulation study of 802.11e based environments with hidden terminals. It points out such flaws of the IEEE 802.11e standard as: disability in providing QoS guarantees within networks with hidden nodes and odd synchronization patterns caused by small $\mathrm{CW}$ sizes for $\mathrm{Vi}$ and Vo traffic. Moreover, the article argues the usefulness of the RTS/CTS mechanism as it did not bring the desired results in both of the analyzed cases. In the presented scenarios, it improves only the throughput of the unhidden station and does not significantly change the throughput levels of the hidden stations. Consequently, it does not remove the meaningful unfairness in granting medium access. The relation between the network performance and the ratio of the hidden to unhidden stations is also stressed.

Even though the presented analysis is thorough, there is still a need of further simulations. Future work will comprise an analysis of new scenarios with varied traffic categories to provide more general conclusions and a new mechanism eliminating the degrading impact of hidden nodes on 802.11e.

\section{ACKNOWLEDGEMENT}

The authors would like to thank OPNET Technologies, Inc. for supporting this research by providing OPNET Modeler software.

\section{REFERENCES}

[1] IEEE 802.11e: Medium Access Control (MAC) Quality of Service Enhancements, New York, IEEE Inc., November 2005

[2] IEEE 802.11b: Higher-speed PHY extension in the $2.4 \mathrm{GHz}$ band, 1999

[3] H. Zhai et al., Medium Access Control in Mobile Ad Hoc Networks: Challenges and Solutions, Wireless Communications and Mobile Computing, vol. 6, pp. 151-170, March 2006

[4] TKN 802.11e module to ns2. [Online] Available: http://www.tkn.tuberlin.de/research/802.11e_ns2

[5] OPNET. [Online] Available: http://www.opnet.com

[6] J. L. Sobrinho et al.: Why RTS-CTS is not your ideal wireless LAN multiple access protocol, vol. 1, pp. 81-87, March 2005

[7] I. Tinnirello, et al: Revisit of RTS/CTS Exchange in High-Speed IEEE 802.11 Networks, pp. 240-248, June 2005

[8] M. Natkaniec and A. R. Pach: Performance Analysis of IEEE 802.11 Networks in the Presence of Hidden Stations, IFIP PWC'2000 Personal Wireless Communications 2000, Poland, pp. 157-168, September 2000 